\title{
Estrogen deficiency and bone loss: an inflammatory tale
}

\author{
M. Neale Weitzmann ${ }^{1}$ and Roberto Pacifici ${ }^{1,2}$
}

1Division of Endocrinology, Metabolism, and Lipids and 2Molecular Pathogenesis Program, Emory University, Atlanta, Georgia, USA.

\begin{abstract}
Estrogen plays a fundamental role in skeletal growth and bone homeostasis in both men and women. Although remarkable progress has been made in our understanding of how estrogen deficiency causes bone loss, the mechanisms involved have proven to be complex and multifaceted. Although estrogen is established to have direct effects on bone cells, recent animal studies have identified additional unexpected regulatory effects of estrogen centered at the level of the adaptive immune response. Furthermore, a potential role for reactive oxygen species has now been identified in both humans and animals. One major challenge is the integration of a multitude of redundant pathways and cytokines, each apparently capable of playing a relevant role, into a comprehensive model of postmenopausal osteoporosis. This Review presents our current understanding of the process of estrogen deficiency-mediated bone destruction and explores some recent findings and hypotheses to explain estrogen action in bone. Due to the inherent difficulties associated with human investigation, many of the lessons learned have been in animal models. Consequently, many of these principles await further validation in humans.
\end{abstract}

The term estrogen refers to numerous steroidal and nonsteroidal molecules capable of inducing estrus. This hormone family plays a fundamental role in skeletal growth and homeostasis. In addition, estrogens are used as pharmacological agents to prevent postmenopausal bone loss. Research during the last decade has revealed that estrogen regulates bone homeostasis through unexpected regulatory effects on the immune system and on oxidative stress and direct effects on bone cells. Many of these observations derive from studies with inbred mice selected for their rapid response to ovariectomy (ovx), which represent an optimal model to investigate the acute effects of estrogen deficiency. However, the conclusions of these studies await confirmation in additional strains of rodents as well as in humans. Since the response to estrogen deprivation is strain specific (1) and estrogen has a more potent anabolic effect in mice than in humans, it is likely that differences will emerge between the mechanisms of estrogen action in humans and rodents.

Prior to 1987, bone cells were not generally considered direct targets of estrogen. However, it is now firmly established that osteoblasts (OBs) (2), osteocytes (3), and osteoclasts (OCs) (4) express functional estrogen receptors (ERs). These receptors are also expressed in bone marrow stromal cells (SCs), the precursors of OBs, which provide physical support for nascent OCs, T cells, B cells, and most other cells in human and mouse bone marrow (5). Estrogen signals through 2 receptors, ER $\alpha$ and ER $\beta$ (6). Bone cells contain both receptors, but their distributions within bone are not homogeneous. In humans, $\mathrm{ER} \alpha$ is the predominant isoform in cortical bone, while ER $\beta$ is the predominant species in trabecular bone. In general, ER $\alpha$ mediates most actions of estrogen on bone cells $(7,8)$. In vitro studies suggest that estrogen's bone-sparing effects are mediated by both estrogen and androgen receptors (9), although subsequent in vivo studies showed that estrogen does

Nonstandard abbreviations used: CIITA, class II transactivator; ER, estrogen receptor; OB, osteoblast; OC, osteoclast; RANK, receptor activator of NF- $\mathrm{BB}$; RANKL,

RANK ligand; ovx, ovariectomy, ovariectomized; SC, stromal cell.

Conflict of interest: The authors have declared that no conflict of interest exists.

Citation for this article: J. Clin. Invest. 116:1186-1194 (2006). doi:10.1172/JCI28550. not prevent bone loss in mice that possess a functional androgen receptor but lack $\operatorname{ER} \alpha$ and $\operatorname{ER} \beta(10)$.

The mechanism through which information is transduced from ligand-bound receptors has been the subject of intense research since 1960. It is now clear that ligand binding to ERs produces a conformational change that promotes receptor dimerization and binding to specific DNA sequences called estrogen response elements (EREs) (11). At the promoter the ligand-bound receptor forms a complex with coactivator proteins, which activates the general transcriptional machinery and increases expression of target genes through chromatin remodeling. ERs can also recruit corepressors, which negatively regulate ER-dependent gene expression. In addition to this classical modality of gene activation, alternative mechanisms have been described that account for estrogen's ability to both stimulate and repress the expression of genes encoding critical osteoclastogenic factors such as IL- 6 , TNF- $\alpha$, and M-CSF. For example, activated ERs can bind to transcription factors such as NF- $\mathrm{BB}$ and prevent binding to DNA, which explains how estrogen represses IL-6 production (12). Equally relevant for the bone-sparing activity of estrogen are its effects on many families of kinases. Estrogen decreases casein kinase 2 (CK2) activity, leading to reduced phosphorylation of the nuclear protein Egr-1. Dephosphorylated Egr-1 has increased affinity for the transcriptional activator Sp-1, a factor critical for expression of the MCSF gene (13). Formation of an Egr-1/Sp-1 complex during estrogen deficiency decreases the nuclear level of free $\mathrm{Sp}-1$, thus blunting MCSF transcription. Estrogen is also capable of blunting JNK activity. The resulting decrease in production of activator protein 1 (AP1) factors explains the repressive effects of estrogen on TNF gene expression (14) as well as why estrogen decreases the sensitivity of maturing OCs to the osteoclastogenic factor receptor activator of NF- $\mathrm{B}$ (RANK) ligand (RANKL) (15).

Although many estrogenic effects are mediated by nuclear ERs, some responses originate in the plasma membrane. In fact, estrogen produces rapid effects (within seconds or minutes) in various cell types, including bone cells. These nongenomic (or nongenotropic) actions are due to signaling by a membrane receptor. Estrogen's ability to induce OC apoptosis and inhibit OB apoptosis is 


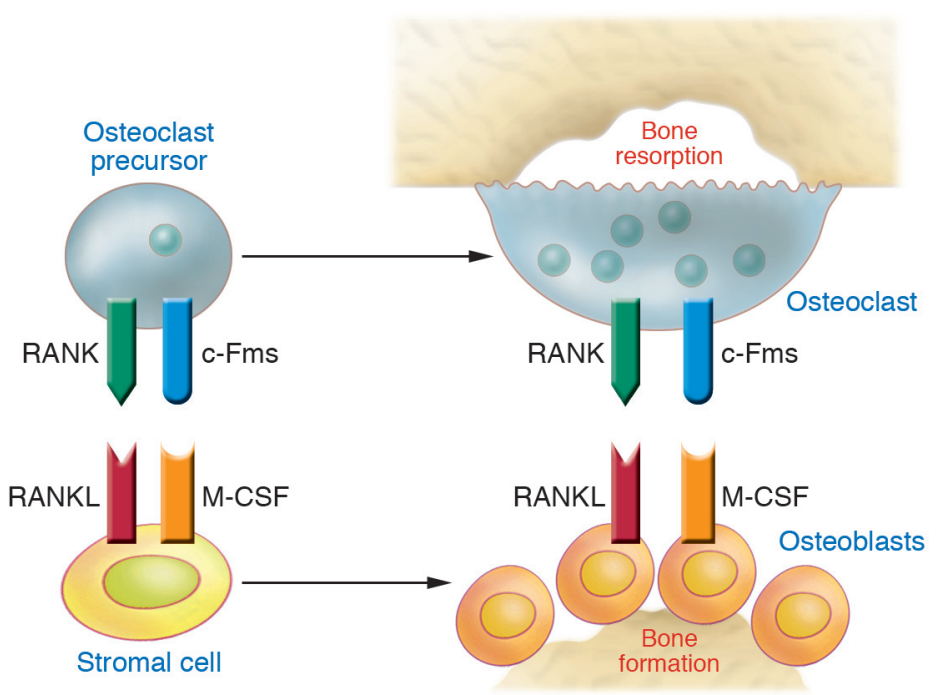

\section{Figure 1}

Cells and cytokines responsible for physiological OC renewal. OC precursors may differentiate from the population of monocytes/macrophages, among which they circulate by virtue of their expression of the receptor RANK. When RANKL binds to this receptor in the presence of the trophic factor $\mathrm{M}-\mathrm{CSF}$, which in turn binds to its receptor, colony-stimulating factor receptor 1 (c-Fms), OC precursors differentiate and fuse together to form mature, multinucleated bone-resorbing OCs. Under physiological conditions the dominant source of RANKL and $\mathrm{M}-\mathrm{CSF}$ in the bone marrow microenvironment is from the bone-forming cells, the OBs, and their SC precursors. linked to its ability to increase ERK1 and ERK2 phosphorylation and repress JNK activity $(9,16)$. The phosphorylation of these cytoplasmic kinases and their transport to the nucleus modulates the activity of transcription factors required for antiapoptotic actions of estrogen (16).

\section{Effects of estrogen deficiency on bone turnover and architecture}

Aging bone is gradually replaced by new tissue through a process called bone remodeling or turnover. Bone remodeling occurs through the coordinated action of OBs and OCs. The activities of OCs and OBs are combined into defined anatomical spaces called basic multicellular units (BMUs) (17). A remodeling cycle begins with the activation of a new BMU on a previously inactive surface of bone. This process involves the disappearance of bone-lining cells and their replacement by OCs that generate resorption lacunae on the endosteal surface of bone over a 2-week interval. The resorption phase is then terminated, probably by OC apoptosis, and after a brief reversal phase, a team of OBs is recruited that fills in the resorption cavity with new bone (18). The net result is the replacement of a packet of old bone with new bone. At menopause there is a transient, accelerated phase of bone loss that is followed by slower, sustained bone loss (18). Although in men there is no abrupt cessation of gonadal function in the sixth decade of life, they do experience an age-related decrease in unbound sex steroids resulting from progressive increases in circulating sex hormone-binding globulin (18). In both men and women there is a steady decline in unbound (bioavailable) estrogen levels with aging, exacerbated in women at menopause by a marked decrease in estrogen levels.

Estrogen deficiency leads to dramatic elevations in the number of BMUs through increased activation frequency, which is the number of new remodeling units activated in each unit of time (19). Enhanced activation frequency expands the remodeling space, increases cortical porosity, and enlarges the resorption area on trabecular surfaces. This phenomenon is caused primarily by increased OC formation, a complex event involving various hematopoietic and immune cells (5), as well as increased OC recruitment to bone surfaces to be remodeled. Estrogen deficiency also augments erosion depth by prolonging the resorption phase of the remodeling cycle through increased OC lifespan due to reduced apoptosis (20).
The net bone loss caused by the combined effects of increased activation frequency and erosion depth is limited in part by a compensatory augmentation of bone formation within each remodeling unit. This event is a consequence of stimulated osteoblastogenesis fueled by an expansion of the pool of early mesenchymal progenitors and by increased commitment of such pluripotent precursors toward the osteoblastic lineage (21). In spite of stimulated osteoblastogenesis, the net increase in bone formation is inadequate to compensate for enhanced bone resorption because of an augmentation in $\mathrm{OB}$ apoptosis, a phenomenon also induced by estrogen deficiency (9).

An additional event triggered by estrogen withdrawal, which limits the magnitude of the compensatory elevation in bone formation, is the increased production of inflammatory cytokines such as IL-7 and TNF, which limit the activity of mature OBs $(22,23)$. Increased bone resorption, trabecular thinning and perforation, and a loss of connection between the remaining trabeculae are the dominant features of the initial phase of rapid bone loss that follows the onset of estrogen deficiency (24). This acute phase is followed by a long-lasting period of slower bone loss where the dominant microarchitectural change is trabecular thinning. This phase is due in part to impaired osteoblastic activity secondary to increased OB apoptosis (25).

Initiation of estrogen replacement therapy (ERT) in experimental animals and humans decreases erosion depth and OC activation frequency by stimulating apoptosis and blocking osteoclastogenesis (19). Long-term ERT at high doses not only blunts bone resorption but also stimulates bone formation, leading to a net anabolic effect (26). A decrease in OB apoptosis resulting from a nongenotropic effect of estrogen is likely a major mechanism driving this effect $(27,28)$. However, the increase in OB lifespan is offset in part by a repressive effect of estrogen on osteoblastogenesis, a phenomenon that explains why the anabolic effect of estrogen is observed only at high doses and during long-term treatment.

\section{Effects of estrogen deficiency on osteoclast formation}

The dominant acute effect of estrogen is the blockade of new OC formation. OCs arise by cytokine-driven proliferation and differentiation of monocyte precursors that circulate within the hematopoietic cell pool (29). This process is facilitated by bone marrow 
SCs, which provide physical support for nascent OCs and produce soluble and membrane-associated factors essential for the proliferation and differentiation of OC precursors (Figure 1).

The minimal essential cytokines required for OC formation under basal conditions are RANKL and M-CSF. These factors are produced primarily by bone marrow SCs, OBs, and activated $\mathrm{T}$ cells (30). RANKL is a TNF superfamily member that exists in membrane-bound and soluble forms. RANKL binds to the transmembrane receptor RANK expressed on the surface of OCs and OC precursors. RANKL also binds to osteoprotegerin (OPG), a soluble decoy receptor produced by numerous hematopoietic cells. Thus OPG, by sequestering RANKL and preventing its binding to RANK, functions as a potent antiosteoclastogenic cytokine (30). RANKL promotes the differentiation of OC precursors from an early stage of maturation into fully mature, multinucleated OCs. RANKL is also capable of activating mature OCs, thus stimulating these cells to resorb bone. M-CSF induces proliferation of early OC precursors, differentiation of more mature OCs, and fusion of mononucleated pre-OCs and increases the survival of mature OCs.

While basal levels of RANKL and M-CSF are essential for physiological OC renewal, additional cytokines either produced or regulated by $\mathrm{T}$ cells are responsible for the upregulation of OC formation observed during estrogen deficiency (5). One such factor is TNF, a cytokine that enhances OC formation directly (31) and by upregulating the SC production of RANKL and the responsiveness of OC precursors to this factor $(32,33)$. The ability of TNF to increase the osteoclastogenic activity of RANKL is due to synergistic interactions at the level of NF-KB and AP1 signaling (34). In addition, TNF and RANKL synergistically upregulate RANK expression in OC precursors (35). Furthermore, TNF stimulates OC activity (36) and inhibits osteoblastogenesis (37), thus further driving an imbalance between bone formation and resorption.

Like TNF, IL-1 promotes RANKL expression by bone marrow SCs and OBs and stimulates OC lifespan and activity. IL-1 directly targets OC precursors and promotes OC differentiation in the presence of permissive levels of RANKL. Furthermore, IL-1 mediates, in part, the osteoclastogenic effect of TNF by enhancing SC expression of RANKL and directly stimulating differentiation of OC precursors (38). TNF and IL-1 have potent antiapoptotic effects in OCs, prolonging OC lifespan and accelerating bone resorption.

\section{T cells and ovariectomy-induced bone loss}

A major focus of osteoporosis research is to understand the reasons for accelerated bone loss following menopause. Thus an important question is how estrogen deficiency leads to increased OC formation. It is recognized that osteoclastogenesis in response to estrogen deficiency is cytokine driven (39). One cytokine responsible for augmented osteoclastogenesis during estrogen deficiency is TNF, and its relevance has been demonstrated in multiple animal models. For example, ovx fails to induce bone loss in TNF KO mice and in mice lacking the p55 TNF receptor (40). Likewise, transgenic mice insensitive to TNF due to the overexpression of a soluble TNF receptor (41) and mice treated with the TNF inhibitor TNF-binding protein (42) are protected from ovx-induced bone loss.

The presence of increased levels of TNF in the bone marrow of ovx animals and in the conditioned media of peripheral blood cells of postmenopausal women is well documented (43-45), although the cells responsible have not been identified conclusively. Recent studies on highly purified bone marrow cells have revealed that ovx increases production of TNF by T cells but not monocytes (33), and that earlier identifications of TNF production by monocytes were likely due to $\mathrm{T}$ cell contamination of monocytes purified by adherence. Thus the ovx-induced increase in TNF levels is likely to be due to $\mathrm{T}$ cell TNF production. These findings in the mouse are concordant with those in humans, in which adherent mononuclear blood cells contain $\mathrm{CD}^{+} \mathrm{CD} 56^{+}$lymphocytes, a TNF-producing subset of adherent $\mathrm{T}$ cells (46). In this study the number of $\mathrm{CD}^{+}$ $\mathrm{CD} 6^{+} \mathrm{T}$ cells was decreased by estrogen treatment and inversely correlated with bone density. These results are not surprising as $\mathrm{T}$ cells can secrete a wide repertoire of cytokines, some pro-osteoclastogenic and some antiosteoclastogenic.

In the absence of strong activation signals, $\mathrm{T}$ cells appear to repress OC formation (47), but the relevance of this phenomenon in vivo has not been established. In contrast, activated $T$ cells play a key role in the regulation of $\mathrm{OC}$ formation through increased production of RANKL and TNF (48-50). Activated T cells also produce IFN- $\alpha$ and IFN- $\gamma$, which in part limit RANKL-induced bone resorption by repressing NF- $\mathrm{KB}$ and JNK signaling pathways (51). The net effect of T cells on OC formation may consequently represent the prevailing balance of pro- and antiosteoclastogenic $\mathrm{T}$ cell cytokine secretion. However, it appears that during stimulated conditions such as inflammation (48) and estrogen deficiency (33), pro-osteoclastogenic cytokines prevail.

Attesting to the relevance of $\mathrm{T}$ cells in estrogen deficiencyinduced bone loss in vivo, measurements of trabecular bone by peripheral quantitative $\mathrm{CT}$ and $\mu \mathrm{CT}$ revealed that athymic T celldeficient nude mice are completely protected from the trabecular bone loss induced by ovx $(33,40,52)$. T cell-deficient mice also fail to respond to ovx with the expected increase in bone turnover $(33,40,52)$. T cells are key inducers of bone wasting because ovx increases $\mathrm{T}$ cell TNF production to a level sufficient to augment RANKL-induced osteoclastogenesis (33). The specific relevance of $\mathrm{T}$ cell TNF production in vivo was demonstrated by the finding that while reconstitution of nude recipient mice with $\mathrm{T}$ cells from wild-type mice restores the capacity of ovx to induce bone loss, reconstitution with $\mathrm{T}$ cells from TNF-deficient mice does not (40). T cell-derived TNF may further augment bone loss by stimulating $\mathrm{T}$ cell RANKL production.

\section{Mechanisms of estrogen regulation of $T$ cell TNF production}

It has been shown that ovx upregulates $\mathrm{T}$ cell TNF production primarily by increasing the number of TNF-producing T cells (40). This is the result of a complex pathway that involves the thymus and bone marrow. The upstream mechanisms by which estrogen deficiency expands the pool of TNF-producing $\mathrm{T}$ cells are summarized in Figure 2. In the bone marrow, ovx promotes $T$ cell activation, resulting in increased $T$ cell proliferation and life span through antigen presentation by macrophages and DCs $(53,54)$. This process is due to the ability of estrogen deficiency to upregulate the expression of MHC class II in macrophages and DCs (53-55). The question thus arises as to the nature of the antigens. Estrogen deficiency is likely to increase $\mathrm{T}$ cell reactivity to a pool of self and foreign antigens physiologically present in healthy animals and humans. This is consistent with the fact that $\mathrm{T}$ cell clones expressing TCRs directed against self antigens not expressed in the thymus survive negative selection during $\mathrm{T}$ cell maturation (56). Such clones (autoreactive or self-reactive $\mathrm{T}$ cells) reside in peripheral lymphatic organs of adult individuals. In addition, foreign antigens of bacterial origin are physiologically absorbed in 


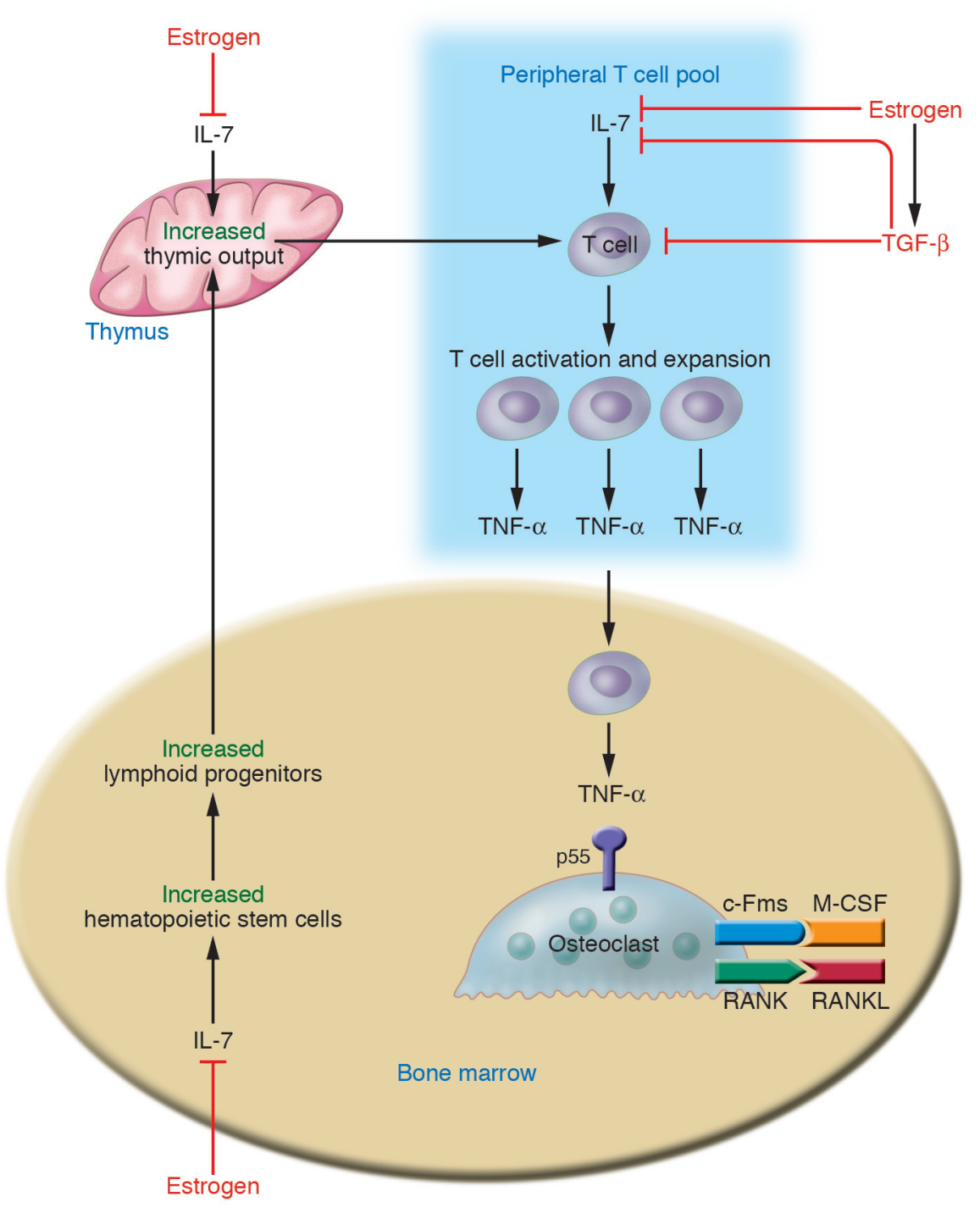

\section{Figure 2}

Estrogen suppresses T cell TNF production by regulating $T$ cell differentiation and activity in the bone marrow, thymus, and peripheral lymphoid organs. In the bone marrow, estrogen downregulates the proliferation of hematopoietic stem cells through an IL-7-dependent mechanism, resulting in a smaller pool of lymphoid progenitors. T cell precursors leave the bone marrow and migrate to the thymus, where $T$ cell differentiation, selection, and expansion take place, in large measure under control of IL-7. Following release from the thymus (thymic output), these new T cells home to peripheral lymphoid organs, including the bone marrow itself. Estrogen prevents $T$ cell activation in part by directly blunting antigen presentation and in part via repression of IL-7 and IFN- $\gamma$ production. This effect is amplified by the upregulation of the IL-7 suppressor TGF- $\beta$. The net result of these actions is a decrease in the number of TNF-producing $T$ cells. The blunted levels of TNF diminish RANKL-induced OC formation, ultimately preventing bone loss. the gut. As these peptides come into contact with immune cells locally and systemically, they induce low-grade $\mathrm{T}$ cell activation (57). Thus a moderate immune response is constantly in place in healthy humans and rodents due to presentation by MHC class II and MHC class I molecules of both self and foreign peptides to $\mathrm{CD}^{+}$and $\mathrm{CD}^{+} \mathrm{T}$ cells (58). This autoreactive response is thought to be essential for immune cell survival and renewal (59).

The effects of ovx on antigen presentation and the resulting changes in $\mathrm{T}$ cell activation, proliferation, and lifespan are explained by a stimulatory effect of ovx on the expression of the gene encoding class II transactivator (CIITA). The product of CIITA is a non-DNA-binding factor induced by IFN- $\gamma$ that functions as a transcriptional coactivator at the MHC class II promoter (60). Increased CIITA expression in macrophages derived from ovx mice results from ovx-mediated increases in both $\mathrm{T}$ cell IFN- $\gamma$ production and the responsiveness of CIITA to IFN- $\gamma$ (53). The relevance of IFN- $\gamma$ to ovx-induced bone loss is suggested by the failure of IFN- $\gamma$ receptor-null mice to undergo $T$ cell activation and sustain bone loss in response to ovx (53).

The actions of IFN- $\gamma$ in bone turnover are controversial. Observations in humans and experimental disease models indicate that
IFN- $\gamma$ promotes bone resorption and causes bone loss under various conditions. For example, IFN- $\gamma$ knockout mice are protected from infection-induced alveolar bone loss (61), while in erosive tuberculoid leprosy and psoriatic arthritis, the number of IFN- $\gamma$ producing cells and the levels of IFN- $\gamma$ in synovial fluid correlates positively with bone destruction $(62,63)$. Furthermore, IFN- $\gamma$ is reported to be efficacious in the treatment of osteopetrosis through restoration of bone resorption in humans (64) and rodents (65). In contrast, others have reported that IFN- $\gamma$ exerts potent antiosteoclastogenic effects in vitro (51), that silencing of IFN- $\gamma$ receptor signaling leads to more rapid onset of collageninduced arthritis and bone resorption (66), and that IFN- $\gamma$ decreases serum calcium concentration and osteoclastic bone resorption in nude mice $(67,68)$.

These conflicting effects of IFN- $\gamma$ may be explained by the fact that IFN- $\gamma$ influences OC formation via both direct and indirect effects. IFN- $\gamma$ directly blocks OC formation through targeting of maturing OCs, as observed in vitro. However, IFN- $\gamma$ is also a potent inducer of antigen presentation and thus of $\mathrm{T}$ cell activation. Therefore, when IFN- $\gamma$ levels are increased in vivo, activated T cells secrete pro-osteoclastogenic factors, and this activity offsets 
the antiosteoclastogenic effect of IFN- $\gamma$. For example, IFN- $\gamma$-producing human $\mathrm{T}$ cells have been reported to directly induce osteoclastogenesis from human monocytes via RANKL expression (69). While under certain conditions the net effect of IFN- $\gamma$ is inhibition of OC formation, other conditions favor stimulation of OC differentiation. Generally, when $T$ cell activation occurs in response to an innate immune response such as LPS exposure, IFN- $\gamma$ functions as an antiresorptive agent. Conversely, when $\mathrm{T}$ cell activation occurs through an adaptive immune response, as in estrogen deficiency, IFN- $\gamma$ stimulates bone resorption. The inability of IFN- $\gamma$ to blunt differentiation of maturing OCs in bone, where RANKL is abundant (70), contributes to the explanation of why in some conditions the in vivo proresorptive effects of IFN- $\gamma$ are more potent than the suppression of osteoclastogenesis induced by IFN- $\gamma$ in vitro.

Estrogen deficiency upregulates IFN- $\gamma$ production through TGF- $\beta$ downregulation. Estrogen has a direct stimulatory effect on the production of this factor, which is mediated through direct binding of the estrogen/ER complex to an ERE in the TGF- $\beta$ promoter (71). TGF- $\beta$ is recognized as a powerful repressor of T cell activation. Indeed, TGF- $\beta$ exerts strong immunosuppressive effects by inhibiting the activation and proliferation of T cells and their production of proinflammatory cytokines including IFN- $\gamma$. Studies in a transgenic mouse model that expresses a dominantnegative form of the TGF- $\beta$ receptor specifically in T cells have contributed to the understanding of the relevance of the repressive effects of this cytokine on $\mathrm{T}$ cell function in bone loss associated with estrogen deficiency (52). This animal model, known as CD4dnTGF $\beta$ RII, is severely osteopenic due to increased bone resorption. More importantly, mice with $\mathrm{T}$ cell-specific blockade of TGF- $\beta$ signaling are completely resistant to the bone-sparing effects of estrogen (52). This phenotype results from a failure of estrogen to repress IFN- $\gamma$ production, which in turn leads to increased $T$ cell activation and TNF production. Gain-of-function experiments confirmed that elevation of the systemic levels of TGF- $\beta$ prevents ovx-induced bone loss and bone turnover (52).

Another mechanism by which estrogen regulates IFN- $\gamma$ and TNF production is by repressing the production of IL-7, a potent lymphopoietic cytokine and inducer of bone destruction in vivo (72). IL-7 receptor knockout mice display increased bone volume and bone mineral density (72). In contrast, IL-7 transgenic mice have expanded bone marrow cavities with focal osteolysis of cortical bone and eroded bone surfaces (73). IL-7 has been reported to induce production of RANKL by human T cells (74), and injection of IL-7 into mice in vivo induces bone destruction by inducing $\mathrm{T}$ cell production of RANKL and TNF (75). Importantly, levels of IL-7 are significantly elevated following ovx $(22,76,77)$, and in vivo IL-7 blockade using neutralizing antibodies is effective in preventing ovx-induced bone destruction (22) by suppressing $\mathrm{T}$ cell expansion and TNF and IFN- $\gamma$ production (76). Furthermore, a recent study shows that liver-derived IGF-1 is permissive for ovxinduced trabecular bone loss by modulation of the number of $\mathrm{T}$ cells and the expression of IL-7 (77). The relevance of IL-7 in the mechanism of ovx-induced bone loss has been confirmed in part by another recent investigation showing that ovx does not induce cortical bone loss in IL-7 knockout mice (78).

Indeed, the elevated bone marrow levels of IL-7 contribute to the expansion of the $\mathrm{T}$ cell population in peripheral lymphoid organs through several mechanisms. First, IL-7 directly stimulates T cell proliferation by lowering tolerance to weak self antigens. Second, IL-7 increases antigen presentation by upregulating the produc- tion of IFN- $\gamma$. Third, IL-7 and TGF- $\beta$ inversely regulate each other's production $(79,80)$. The reduction in TGF- $\beta$ signaling, characteristic of estrogen deficiency, may serve to further stimulate IL-7 production, thus driving the cycle of osteoclastogenic cytokine production and bone wasting. New studies further implicate IL-7 as a downstream effector of IGF-1 action in ovx-induced trabecular bone loss (77).

In estrogen deficiency, IL-7 compounds bone loss by suppressing bone formation, thus uncoupling bone formation from resorption. Recent studies have also identified elevated levels of IL-7 in patients suffering from multiple myeloma and in multiple myeloma-derived cell lines (81) and have suggested a role for IL-7 in the enhanced bone resorption and suppressed bone formation associated with multiple myeloma. Increased IL-7 expression has also been implicated in the bone loss sustained by patients with rheumatoid arthritis $(82,83)$.

IL-7 is a stimulator of both B and T cell lineages, and it has been suggested that IL-7 also induces bone loss by a mechanism involving the expansion of cells of the B lineage, in particular B220+ $\operatorname{IgM}^{-}$ $\mathrm{B}$ cell precursors (72), a population that is greatly expanded during estrogen deficiency $(72,84)$. How B lineage cells may lead to bone destruction is not presently understood but may involve overexpression of RANKL, a property of activated B cells (85). Alternatively, early B220+ $\mathrm{IgM}^{-}$precursor cells have been found to be capable of differentiating into OCs in response to M-CSF and/ or RANKL in vitro $(75,86)$ and may thus contribute to increasing the pool of early OC precursors. These cells have likewise been suggested to play a potential role in arthritic bone destruction.

\section{T cell thymic output and bone loss}

The thymus undergoes progressive structural and functional decline with age, coinciding with increased circulating sex steroid levels at puberty (87). By middle age most parenchymal tissue is replaced by fat, and in both mice and humans fewer $T$ cells are produced and exported to secondary lymphoid organs. However, the thymus continues to generate new $\mathrm{T}$ cells even into old age (88, 89). In fact, active lymphocytic thymic tissue has been documented in adults up to 107 years of age (90). Under severe T cell depletion secondary to HIV infection, chemotherapy, or bone marrow transplant, an increase in thymic output (known as thymic rebound) becomes critical for long-term restoration of $\mathrm{T}$ cell homeostasis. For example, middle-aged women treated with autologous bone marrow transplants develop thymic hypertrophy and a resurgence of thymic T cell output, which contributes to the restoration of a wide $T$ cell repertoire (91), although the intensity of thymic rebound declines with age. The mechanism driving thymic rebound is not completely understood, but one factor involved is IL-7 (92). Importantly, IL-7 alone is not sufficient to enhance thymopoiesis in young mice (93) but plays a more relevant role in aged mice (94).

Both androgens and estrogen have a profound suppressive effect on thymic function. Accordingly, castration reverses thymic atrophy and increases export of recent thymic emigrants to the periphery (95), while sex steroid inhibits thymus regeneration by promoting thymocyte apoptosis and an arrest of differentiation (96). Restoration of thymic function after castration occurs in young (97) as well as in very old rodents (98).

In accordance with the notion that estrogen deficiency induces a rebound in thymic function, ovx expands the population of thymic $\mathrm{T}$ cells and leads to the thymic export of naive T cells (76). Indeed, stimulated thymic $\mathrm{T}$ cell output accounts for approximately $50 \%$ 


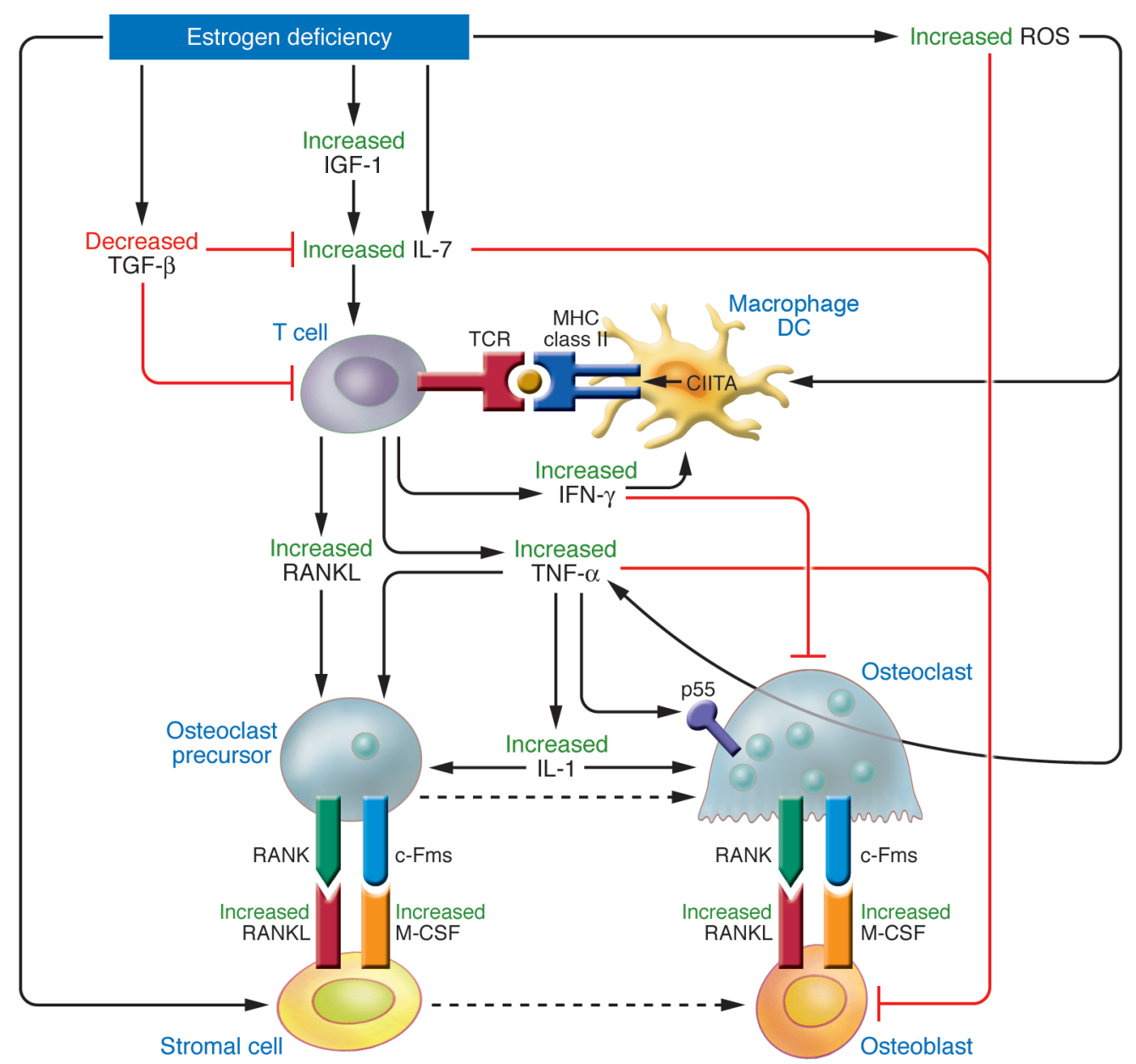

Figure 3

Schematic representation of the main mechanisms and feedback interactions by which estrogen deficiency leads to bone loss. The bone loss induced by estrogen deficiency is due to a complex interplay of hormones and cytokines that converge to disrupt the process of bone remodeling. Estrogen deficiency leads to a global increase in IL-7 production in target organs such as bone, thymus, and spleen, in part through decreases in TGF- $\beta$ and increased IGF-1 production. This leads to an initial wave of T cell activation. Activated T cells release IFN- $\gamma$, which increases antigen presentation by $\mathrm{DC}$ and macrophages $(\mathrm{M} \phi)$ by upregulating $\mathrm{MHC}$ class II expression through the transcription factor CIITA. Estrogen deficiency also amplifies T cell activation and osteoclastogenesis by downregulating antioxidant pathways, leading to an upswing in ROS. The resulting increase in ROS stimulates antigen presentation and the production of TNF by mature OCs. The combined effect of IFN- $\gamma$ and ROS markedly enhances antigen presentation, amplifying $T$ cell activation and promoting release of the osteoclastogenic factors RANKL and TNF. TNF further stimulates SC and OB RANKL and M-CSF production, in part via IL-1 upregulation, driving OC formation. TNF and IL-7 further exacerbate bone loss by blunting bone formation through direct repressive effects on OBs.

of the increase in the number of T cells in the periphery, while the remainder is due to enhanced peripheral expansion. Similarly, thymectomy reduces by approximately $50 \%$ the bone loss induced by ovx, thus demonstrating that the thymus plays a previously unrecognized causal effect in ovx-induced bone loss in mice. The remaining bone loss is a consequence of the peripheral expansion of naive and memory T cells (76). This finding, which awaits confirmation in humans, suggests that estrogen deficiency-induced thymic rebound may be responsible for the exaggerated bone loss in young women undergoing surgical menopause (99) or for the rapid bone loss characteristic of women in their first 5-7 years after natural menopause (18). Indeed, an age-related decrease in estrogen deficiency-induced thymic rebound could mitigate the stimulatory effects of sex steroid deprivation and explain why the rate of bone loss in postmenopausal women diminishes as aging progresses (18).

\section{Estrogen, oxidative stress, and T cell-dependent bone loss}

Recently, it has been suggested that ROS may play a role in postmenopausal bone loss by generating a more oxidized bone microenvironment $(100,101)$. In vivo support of this hypothesis is found from experiments in which ovx induces oxidative stress and impairs antioxidant expression in adult rats (102). Furthermore, administration of agents that increase the intracellular concentration of the antioxidant glutathione in bone prevents bone loss during estrogen deficiency in mice, while depletion of glutathione by buthionine sulfoximine (BSO), which inhibits glutathione synthesis, enhances bone loss (103). The NO donor nitroglycerin is also reported to prevent bone loss in ovx rats $(104,105)$, while in the presence of $\mathrm{N}$-nitro-l-arginine methyl ester (L-NAME), an NO synthase inhibitor, estrogen was ineffective in reversing bone loss. This suggests that 
the protective effect of estrogen may be mediated in part through NO (104). In human studies nitroglycerin significantly prevented osteoporotic fractures in postmenopausal women (106).

The mechanisms of action of ROS and the cellular targets that regulate bone mass are poorly understood. OCs have been shown to both generate and be activated by $\operatorname{ROS}(107,108)$. Glutathione peroxidase, responsible for intracellular degradation of hydrogen peroxide, is the predominant antioxidant enzyme expressed by OCs (109) and is upregulated by estrogen. Overexpression of glutathione peroxidase in the preosteoclastic cell line RAW 264.7 abolishes OC formation (109). This suppression of osteoclastic differentiation by antioxidants is likely to occur through protection of phosphatases from reversible inhibition by the ROS hydrogen peroxide (110) and by suppression of thioredoxin expression. Thioredoxin is induced by oxidative stress and enhances OC formation (111). Consistent with a role for hydrogen peroxide in this pathway, catalase was found to prevent ovx-induced bone loss in mice (109).

Estrogen enhances the levels of antioxidants in many cell lineages including the OC $(103,112)$. The expression of OC TNF is augmented by ROS. Bone loss caused by BSO has significant similarities to bone loss induced by estrogen deficiency, as both processes are TNF dependent (113). Moreover, soluble TNF receptors prevent both bone loss and the rise in thiol-based antioxidants characteristic of estrogen deficiency (113).

Although the mechanisms of ROS action on bone during estrogen deficiency are poorly understood, it is known that immune cells are biological targets of ROS. ROS are important stimulators of antigen presentation by DCs as well as DC-induced T cell activation. Antioxidants potently inhibit DC differentiation and activation of $\mathrm{T}$ cells $(114,115)$ in part by suppressing expression of MHC class II and costimulatory molecules in response to antigen (116). $N$-acetyl-cysteine (NAC), which acts as an intracellular scavenger by restoring intracellular concentration of glutathione, can block DC maturation (117) and DC-mediated T cell activation (118). ROS are also generated upon DC interaction with $\mathrm{T}$ cells (119) and can reduce $\mathrm{T}$ cell lifespan by stimulating $\mathrm{T}$ cell apoptosis (120). Interestingly, NAC treatment has been shown to protect against ovx-induced bone loss (103). These data are consistent with studies demonstrating that NAC treatment blunts ovx-induced DC activation in the bone marrow, decreases antigen presentation and expression of costimulatory molecules, and prevents $\mathrm{T}$ cell activation and TNF production (121). Taken together, these data suggest a model for ovx-induced bone loss in which estrogen deficiency lowers antioxidant levels, thereby increasing ROS. Additionally, estrogen deficiency augments TNF expression by enhancing OC-mediated TNF production and by stimulating APC-induced expansion of the TNF-producing $\mathrm{T}$ cells that are central to bone destruction.

\section{Conclusions}

Remarkable progress has been made in the last 2 decades in our understanding of the mechanisms of bone destruction during estrogen deficiency. The directions that future research into postmenopausal osteoporosis will take are hard to predict, and new surprises are likely in store. For example, an intriguing link has recently been made between the sympathetic nervous system and bone loss during gonadal failure (122). Our view of postmenopausal osteoporosis will no doubt continue to evolve over the next decade, and radical new therapies will ultimately follow as we gain new knowledge and understanding of this multifaceted malady.
Most new data are derived from studies in mice and remain to be validated in humans. These validation studies will be essential for defining the role of inflammatory cytokines in postmenopausal bone loss, as selective inhibitors might be developed as new therapeutic agents.

The ovx mouse is an excellent model to investigate the acute effects of estrogen withdrawal, although it is not suitable to study the long-term skeletal effects of menopause, as bone loss subsides within a few weeks after ovx in this model. Thus additional animal models and long-term human studies are needed. Since critical effects of estrogen on bone involve regulation of precursor cell differentiation and signaling pathways, which are few and short-lived, many pivotal effects of estrogen in vivo are difficult to reproduce in vitro. Similarly, regulatory events observed in vitro are often not relevant in vivo. It is therefore essential that in vitro studies are validated using in vivo model systems. For example, while estrogen stimulates IFN- $\gamma$ production in cell cultures (122), estrogen represses it in vivo (53). Similarly, while IFN- $\gamma$ blocks OC formation through direct targeting of maturing OCs, IFN- $\gamma$ stimulates osteoclastogenesis and bone resorption in estrogen-deficient mice.

In summary, the multifaceted activities of estrogen are fully reflected in bone. Of the many surprises encountered investigating estrogen action in bone is the relationship among estrogen, the immune system, and the skeleton (Figure 3). Clearly, if this relationship is equally relevant in humans as in rodents, postmenopausal osteoporosis should be regarded as the product of an inflammatory disease bearing many characteristics of an organlimited autoimmune disorder, triggered by estrogen deficiency, and brought about by chronic mild decreases in $\mathrm{T}$ cell tolerance. Why such a pathway should have emerged is intriguing. One explanation is suggested by the need to stimulate bone resorption in the immediate postpartum period in order to meet the markedly increased maternal demand for calcium brought about by milk production. The signal for this event is the drop in estrogen levels early postpartum. Henry Kronenberg (Harvard University, Boston, Massachusetts, USA) has suggested that postmenopausal bone loss should be regarded as an unintended recapitulation of this phenomenon (personal communication).

Another response to delivery is the restoration of normal immune reactivity and the loss of tolerance to the fetus. It is tempting to speculate that cessation of ovarian function induces bone loss through an adaptive immune response because natural selection has centralized these 2 key adaptations to postpartum within the immune system.

\section{Acknowledgments}

M.N. Weitzmann is supported in part by grants from the National Osteoporosis Foundation and the National Institutes of Diabetes and Digestive and Kidney Diseases (DK067389). R. Pacifici is supported in part by grants from the National Institutes of Health (AR 49659). We are grateful to Francesco Grassi (Emory University, Atlanta, Georgia, USA) and Timothy Chambers (St. George's Hospital Medical School, London, United Kingdom) for their helpful suggestions.

Address correspondence to: Roberto Pacifici, Division of Endocrinology, Metabolism, and Lipids, Emory University School of Medicine, 101 Woodruff Circle, Room 1307, Atlanta, Georgia 30322, USA. Phone: (404) 712-8420; Fax: (404) 727-1300; E-mail: roberto.pacifici@emory.edu. 
1. Bouxsein, M.L., et al. 2005. Ovariectomy-induced bone loss varies among inbred strains of mice. J. Bone Miner. Res. 20:1085-1092.

2. Komm, B.S., et al. 1988. Estrogen binding, receptor mRNA, and biologic response in osteoblast-like osteosarcoma cells. Science. 241:81-84.

3. Tomkinson, A., Gevers, E.F., Wit, J.M., Reeve, J., and Noble, B.S. 1998. The role of estrogen in the control of rat osteocyte apoptosis. J. Bone Miner. Res. 13:1243-1250.

4. Oursler, M.J., Osdoby, P., Pyfferoen, J., Riggs, B.L., and Spelsberg, T.C. 1991. Avian osteoclasts as estrogen target cells. Proc. Natl. Acad. Sci. U. S. A. 88:6613-6617.

5. Weitzmann, M.N., and Pacifici, R. 2005. The role of T lymphocytes in bone metabolism. Immunol. Rev 208:154-168

6. Kuiper, G.G., Enmark, E., Pelto-Huikko, M., Nilsson, S., and Gustafsson, J.A. 1996. Cloning of a novel receptor expressed in rat prostate and ovary. Proc. Natl. Acad. Sci. U. S. A. 93:5925-5930.

7. Barkhem, T., et al. 1998. Differential response of estrogen receptor alpha and estrogen receptor beta to partial estrogen agonists/antagonists. Mol. Pharmacol. 54:105-112.

8. Hall, J.M., and McDonnell, D.P. 1999. The estrogen receptor beta-isoform (ERbeta) of the human estrogen receptor modulates ERalpha transcriptional activity and is a key regulator of the cellular response to estrogens and antiestrogens. Endocrinology. 140:5566-5578.

9. Kousteni, S., et al. 2001. Nongenotropic, sex-nonspecific signaling through the estrogen or androgen receptors: dissociation from transcriptional activity. Cell. 104:719-730.

10. Sims, N.A., et al. 2003. A functional androgen receptor is not sufficient to allow estradiol to protect bone after gonadectomy in estradiol receptor-deficient mice. J. Clin. Invest. 111:1319-1327. doi:10.1172/JCI200317246.

11. Smith, C.L., and O'Malley, B.W. 2004. Coregulator function: a key to understanding tissue specificity of selective receptor modulators. Endocr. Rev. 25:45-71.

12. Stein, B., and Yang, M.X. 1995. Repression of the interleukin- 6 promoter by estrogen receptor is mediated by NF-kappa B and C/EBP beta. Mol. Cell. Biol. 15:4971-4979.

13. Srivastava, S., et al. 1998. Estrogen blocks M-CSF gene expression and osteoclast formation by regulating phosphorylation of Egr-1 and its interaction with Sp-1. J. Clin. Invest. 102:1850-1859.

14. Srivastava, S., et al. 1999. Estrogen decreases TNF gene expression by blocking JNK activity and the resulting production of c-jun and junD. J. Clin. Invest. 104:503-513

15. Srivastava, S., et al. 2001. Estrogen decreases osteoclast formation by down-regulating receptor activator of NF-kappa B ligand (RANKL)-induced JNK activation. J. Biol. Chem. 276:8836-8840.

16. Kousteni, S., et al. 2003. Kinase-mediated regulation of common transcription factors accounts for the bone-protective effects of sex steroids. J. Clin. Invest. 111:1651-1664. doi:10.1172/JCI200317261.

17. Frost, H.M. 1983. Bone histomorphometry: analysis of trabecular bone dynamics. In Bone histomorphometry: techniques and interpretation. R.R. Recker, editor. CRC Press. Boca Raton, Florida, USA. 109-131.

18. Riggs, B.L., Khosla, S., and Melton, L.J., 3rd. 2002. Sex steroids and the construction and conservation of the adult skeleton. Endocr. Rev. 23:279-302.

19. Eriksen, E.F., Langdahl, B., Vesterby, A., Rungby, J., and Kassem, M. 1999. Hormone replacement therapy prevents osteoclastic hyperactivity: a histomorphometric study in early postmenopausal women. J. Bone Miner. Res. 14:1217-1221.

20. Hughes, D.E., et al. 1996. Estrogen promotes apoptosis of murine osteoclasts mediated by TGF-beta. Nat. Med. 2:1132-1136.

21. Jilka, R.L., et al. 1998. Loss of estrogen upregulates osteoblastogenesis in the murine bone marrow. Evidence for autonomy from factors released during bone resorption. J. Clin. Invest. 101:1942-1950.

22. Weitzmann, M.N., Roggia, C., Toraldo, G., Weitzmann, L., and Pacifici, R. 2002. Increased production of IL-7 uncouples bone formation from bone resorption during estrogen deficiency. J. Clin. Invest. 110:1643-1650. doi:10.1172/JCI200317261.

23. Gilbert, L., et al. 2000. Inhibition of osteoblast differentiation by tumor necrosis factor-alpha. Endocrinology. 141:3956-3964.

24. Eriksen, E.F., et al. 1990. Cancellous bone remodeling in type I (postmenopausal) osteoporosis: quantitative assessment of rates of formation, resorption, and bone loss at tissue and cellular levels. J. Bone Miner. Res. 5:311-319.

25. Riggs, B.L., and Parfitt, A.M. 2005. Drugs used to treat osteoporosis: the critical need for a uniform nomenclature based on their action on bone remodeling. J. Bone Miner. Res. 20:177-184.

26. Vedi, S., et al. 1999. Bone remodeling and structure in postmenopausal women treated with long-term, high-dose estrogen therapy. Osteoporos. Int. 10:52-58.

27. Manolagas, S.C. 2000. Birth and death of bone cells: basic regulatory mechanisms and implications for the pathogenesis and treatment of osteoporosis. Endocr. Rev. 21:115-137.

28. Manolagas, S.C., Kousteni, S., and Jilka, R.L. 2002. Sex steroids and bone. Recent Prog. Horm. Res. 57:385-409.

29. Teitelbaum, S.L. 2000. Bone resorption by osteoclasts. Science. 289:1504-1508.

30. Khosla, S. 2001. Minireview: the OPG/RANKL/ RANK system. Endocrinology. 142:5050-5055.

31. Kim, N., et al. 2005. Osteoclast differentiation independent of the TRANCE-RANK-TRAF6 axis. J. Exp. Med. 202:589-595.

32. Hofbauer, L.C., et al. 1999. Interleukin-1beta and tumor necrosis factor-alpha, but not interleukin-6, stimulate osteoprotegerin ligand gene expression in human osteoblastic cells. Bone. 25:255-259.

33. Cenci, S., et al. 2000. Estrogen deficiency induces bone loss by enhancing T-cell production of TNF- $\alpha$. J. Clin. Invest. 106:1229-1237.

34. Lam, J., et al. 2000. TNF- $\alpha$ induces osteoclastogenesis by direct stimulation of macrophages exposed to permissive levels of RANK ligand. J. Clin. Invest. 106:1481-1488.

35. Zhang, Y.H., Heulsmann, A., Tondravi, M.M., Mukherjee, A., and Abu-Amer, Y. 2001. Tumor necrosis factor-alpha (TNF) stimulates RANKL-induced osteoclastogenesis via coupling of TNF type 1 receptor and RANK signaling pathways. J. Biol. Chem. 276:563-568

36. Fuller, K., Murphy, C., Kirstein, B., Fox, S.W., and Chambers, T.J. 2002. TNFalpha potently activates osteoclasts, through a direct action independent of and strongly synergistic with RANKL. Endocrinology. 143:1108-1118.

37. Nanes, M.S. 2003. Tumor necrosis factor-alpha: molecular and cellular mechanisms in skeletal pathology. Gene. 321:1-15.

38. Wei, S., Kitaura, H., Zhou, P., Ross, F.P., and Teitelbaum, S.L. 2005. IL-1 mediates TNF-induced osteoclastogenesis. J. Clin. Invest. 115:282-290. doi:10.1172/JCI200317261.

39. Pfeilschifter, J., Koditz, R., Pfohl, M., and Schatz, H. 2002. Changes in proinflammatory cytokine activity after menopause. Endocr. Rev. 23:90-119.

40. Roggia, C., et al. 2001. Up-regulation of TNF-producing $\mathrm{T}$ cells in the bone marrow: a key mechanism by which estrogen deficiency induces bone loss in vivo. Proc. Natl. Acad. Sci. U. S. A. 98:13960-13965.

41. Ammann, P., et al. 1997. Transgenic mice expressing soluble tumor necrosis factor-receptor are protected against bone loss caused by estrogen deficiency. J. Clin. Invest. 99:1699-1703.

42. Kimble, R., Bain, S., and Pacifici, R. 1997. The functional block of TNF but not of IL- 6 prevents bone loss in ovariectomized mice. J. Bone Min. Res. 12:935-941.

43. Pacifici, R., et al. 1991. Effect of surgical menopause and estrogen replacement on cytokine release from human blood mononuclear cells. Proc. Natl. Acad. Sci. U. S. A. 88:5134-5138.

44. Ralston, S.H., Russell, R.G.G., and Gowen, M. 1990. Estrogen inhibits release of tumor necrosis factor from peripheral blood mononuclear cells in postmenopausal women. J. Bone Miner. Res. 5:983-988.

45. Shanker, G., Sorci-Thomas, M., and Adams, M.R. 1994. Estrogen modulates the expression of tumor necrosis factor alpha mRNA in phorbol ester-stimulated human monocytic THP-1 cells. Lymphokine Cytokine Res. 13:377-382.

46. Abrahamsen, B., Bendtzen, K., and Beck-Nielsen, H. 1997. Cytokines and T-lymphocyte subsets in healthy post-menopausal women: estrogen retards bone loss without affecting the release of IL-1 or IL-1ra. Bone. 20:251-258.

47. Grcevic, D., Lee, S.K., Marusic, A., and Lorenzo, J.A. 2000. Depletion of CD4 and CD8 T lymphocytes in mice in vivo enhances 1,25 - dihydroxyvitamin $\mathrm{D}(3)$-stimulated osteoclast-like cell formation in vitro by a mechanism that is dependent on prostaglandin synthesis. J. Immunol. 165:4231-4238.

48. Kong, Y.Y., et al. 1999. Activated T cells regulate bone loss and joint destruction in adjuvant arthritis through osteoprotegerin ligand. Nature. 402:304-309.

49. Horwood, N.J., et al. 1999. Activated T lymphocytes support osteoclast formation in vitro. Biochem. Biophys. Res. Commun. 265:144-150.

50. Weitzmann, M.N., et al. 2001. T cell activation induces human osteoclast formation via receptor activator of nuclear factor kappaB ligand-dependent and -independent mechanisms. J. Bone Miner. Res. 16:328-337.

51. Takayanagi, H., et al. 2000. T-cell-mediated regulation of osteoclastogenesis by signalling crosstalk between RANKL and IFN-gamma. Nature. 408:600-605.

52. Gao, Y., et al. 2004. Estrogen prevents bone loss through transforming growth factor beta signaling in T cells. Proc. Natl. Acad. Sci. U. S. A. 101:16618-16623.

53. Cenci, S., et al. 2003. Estrogen deficiency induces bone loss by increasing $\mathrm{T}$ cell proliferation and lifespan through IFN-gamma-induced class II transactivator. Proc. Natl. Acad. Sci. U. S. A. 100:10405-10410.

54. Grassi, F., and Pacifici, R. 2005. Ovariectomy increases the formation of $\mathrm{T}$ cell niches at the resorption surfaces. J. Bone Miner. Res. 20:Abs F395.

55. Adamski, J., Ma, Z., Nozell, S., and Benveniste, E.N. 2004. 17beta-Estradiol inhibits class II major histocompatibility complex (MHC) expression: influence on histone modifications and $\mathrm{cbp}$ recruitment to the class II MHC promoter. Mol. Endocrinol. 18:1963-1974.

56. Robey, E.A., et al. 1992. A self-reactive T cell population that is not subject to negative selection. Int. Immunol. 4:969-974.

57. Rammensee, H.G., Falk, K., and Rotzschke, O. 1993. Peptides naturally presented by MHC class I molecules. Annu. Rev. Immunol. 11:213-244.

58. Grossman, Z., and Paul, W.E. 2000. Self-tolerance: context dependent tuning of $\mathrm{T}$ cell antigen recognition. Semin. Immunol. 12:197-203; discussion 257-344.

59. Tanchot, C., Lemonnier, F.A., Perarnau, B., Freitas, A.A., and Rocha, B. 1997. Differential requirements for survival and proliferation of CD8 naive or memory T cells. Science. 276:2057-2062.

60. Boss, J.M., and Jensen, P.E. 2003. Transcriptional regulation of the MHC class II antigen presentation pathway. Curr. Opin. Immunol. 15:105-111.

61. Baker, P.J., et al. 1999. CD4(+) T cells and the proinflammatory cytokines gamma interferon and interleukin- 6 contribute to alveolar bone loss in mice. Infect. Immun. 67:2804-2809. 
62. Arnoldi, J., Gerdes, J., and Flad, H.D. 1990. Immunohistologic assessment of cytokine production of infiltrating cells in various forms of leprosy. Am.J. Pathol. 137:749-753.

63. Firestein, G.S., Alvaro-Gracia,J.M., and Maki, R.. 1990. Quantitative analysis of cytokine gene expression in rheumatoid arthritis. J. Immunol. 144:3347-3353.

64. Key, L.L., Jr., et al. 1995. Long-term treatment of osteopetrosis with recombinant human interferon gamma. N. Engl. J. Med. 332:1594-1599.

65. Rodriguiz, R.M., Key, L.L., Jr., and Ries, W.L. 1993. Combination macrophage-colony stimulating factor and interferon-gamma administration ameliorates the osteopetrotic condition in microphthalmic (mi/mi) mice. Pediatr. Res. 33:384-389.

66. Vermeire, K., et al. 1997. Accelerated collageninduced arthritis in IFN-gamma receptor-deficient mice. J. Immunol. 158:5507-5513.

67. Sato, K., et al. 1992. Prolonged decrease of serum calcium concentration by murine gamma-interferon in hypercalcemic, human tumor (EC-GI)-bearing nude mice. Cancer Res. 52:444-449.

68. Tohkin, M., Kakudo, S., Kasai, H., and Arita, H. 1994. Comparative study of inhibitory effects by murine interferon gamma and a new bisphosphonate (alendronate) in hypercalcemic, nude mice bearing human tumor (LJC-1-JCK). Cancer Immunol. Immunother. 39:155-160.

69. Kotake, S., et al. 2005. IFN-gamma-producing human $\mathrm{T}$ cells directly induce osteoclastogenesis from human monocytes via the expression of RANKL. Eur. J. Immunol. 35:3353-3363.

70. Huang, W., O'Keefe, R.J., and Schwarz, E.M. 2003. Exposure to receptor-activator of NFkappaB ligand renders pre-osteoclasts resistant to IFN-gamma by inducing terminal differentiation. Arthritis. Res. Ther. 5:R49-R59.

71. Yang, N.N., Venugopalan, M., Hardikar, S., and Glasebrook, A. 1996. Identification of an estrogen response element activated by metabolites of $17 \mathrm{~b}$ estradiol and raloxifene. Science. 273:1222-1225.

72. Miyaura, C., et al. 1997. Increased B-lymphopoiesis by interleukin 7 induces bone loss in mice with intact ovarian function: similarity to estrogen deficiency. Proc. Natl. Acad. Sci. U. S. A. 19:9360-9365.

73. Valenzona, H.O., Pointer, R., Ceredig, R., and Osmond, D.G. 1996. Prelymphomatous B cell hyperplasia in the bone marrow of interleukin-7 transgenic mice: precursor B cell dynamics, microenvironmental organization and osteolysis. Exp. Hematol. 24:1521-1529.

74. Weitzmann, M.N., Cenci, S., Rifas, L., Brown, C., and Pacifici, R. 2000. Interleukin-7 stimulates osteoclast formation by up-regulating the T-cell production of soluble osteoclastogenic cytokines. Blood. 96:1873-1878.

75. Toraldo, G., Roggia, C., Qian, W.P., Pacifici, R., and Weitzmann, M.N. 2003. IL-7 induces bone loss in vivo by induction of receptor activator of nuclear factor kappa $B$ ligand and tumor necrosis factor alpha from T cells. Proc. Natl. Acad. Sci. U. S. A. 100:125-130.

76. Ryan, M.R., et al. 2005. An IL-7-dependent rebound in thymic $\mathrm{T}$ cell output contributes to the bone loss induced by estrogen deficiency. Proc. Natl. Acad. Sci. U. S. A. 102:16735-16740.

77. Lindberg, M.K., et al. 2006. Liver-derived IGF-I is permissive for ovariectomy-induced trabecular bone loss. Bone. 38:85-92

78. Lee, S.K., et al. 2006. Interleukin-7 influences osteoclast function in vivo but is not a critical factor in ovariectomy-induced bone loss. J. Bone Miner. Res. doi: $10.1359 /$ jbmr.060117.

79. Huang, M., et al. 2002. IL-7 inhibits fibroblast TGF- $\beta$ production and signaling in pulmonary fibrosis. $J$. Clin. Invest. 109:931-937. doi:10.1172/JCI200214685.

80. Dubinett, S.M., et al. 1995. Down-regulation of murine fibrosarcoma transforming growth factorbeta 1 expression by interleukin 7. J. Natl. Cancer
Inst. 87:593-597.

81. Giuliani, N., et al. 2002. Human myeloma cells stimulate the receptor activator of nuclear factor-kappa B ligand (RANKL) in T lymphocytes: a potential role in multiple myeloma bone disease. Blood. 100:4615-4621

82. van Roon, J.A., Glaudemans, K.A., Bijlsma, J.W., and Lafeber, F.P. 2003. Interleukin 7 stimulates tumour necrosis factor alpha and Th1 cytokine production in joints of patients with rheumatoid arthritis. Ann. Rheum. Dis. 62:113-119.

83. De Benedetti, F., et al. 1995. Elevated circulating interleukin-7 levels in patients with systemic juvenile rheumatoid arthritis. J. Rheumatol. 22:1581-1585.

84. Masuzawa, T., et al. 1994. Estrogen deficiency stimulates B lymphopoiesis in mouse bone marrow. J. Clin. Invest. 94:1090-1097.

85. Manabe, N., et al. 2001. Connection between B lymphocyte and osteoclast differentiation pathways. J. Immunol. 167:2625-2631.

86. Sato, T., Shibata, T., Ikeda, K., and Watanabe, K. 2001. Generation of bone-resorbing osteoclasts from B220+ cells: its role in accelerated osteoclastogenesis due to estrogen deficiency. J. Bone Miner. Res. 16:2215-2221.

87. Haynes, B.F., Sempowski, G.D., Wells, A.F., and Hale, L.P. 2000. The human thymus during aging. Immunol. Res. 22:253-261.

88. Douek, D.C., and Koup, R.A. 2000. Evidence for thymic function in the elderly. Vaccine. 18:1638-1641.

89. Jamieson, B.D., et al. 1999. Generation of functional thymocytes in the human adult. Immunity. 10:569-575.

90. Steinmann, G.G., Klaus, B., and Muller-Hermelink, H.K. 1985. The involution of the ageing human thymic epithelium is independent of puberty. A morphometric study. Scand. J. Immunol. 22:563-575.

91. Hakim, F.T., et al. 2005. Age-dependent incidence, time course, and consequences of thymic renewal in adults. J. Clin. Invest. 115:930-939. doi:10.1172/ JCI200317261.

92. Mackall, C.L., et al. 2001. IL-7 increases both thymic-dependent and thymic-independent T-cell regeneration after bone marrow transplantation. Blood. 97:1491-1497.

93. Chu, Y.W., et al. 2004. Exogenous IL-7 increases recent thymic emigrants in peripheral lymphoid tissue without enhanced thymic function. Blood. 104:1110-1119.

94. Alpdogan, O., et al. 2001. Administration of interleukin-7 after allogeneic bone marrow transplantation improves immune reconstitution without aggravating graft-versus-host disease. Blood. 98:2256-2265.

95. Utsuyama, M., and Hirokawa, K. 1989. Hypertrophy of the thymus and restoration of immune functions in mice and rats by gonadectomy. Mech. Ageing Dev. 47:175-185.

96. Okasha, S.A., et al. 2001. Evidence for estradiolinduced apoptosis and dysregulated $\mathrm{T}$ cell maturation in the thymus. Toxicology. 163:49-62.

97. Roden, A.C., et al. 2004. Augmentation of T cell levels and responses induced by androgen deprivation. J. Immunol. 173:6098-6108.

98. Sutherland, J.S., et al. 2005. Activation of thymic regeneration in mice and humans following androgen blockade. J. Immunol. 175:2741-2753.

99. Hreshchyshyn, M.M., Hopkins, A., Zylstra, S., and Anbar, M. 1988. Effects of natural menopause, hysterectomy, and oophorectomy on lumbar spine and femoral neck bone densities. Obstet. Gynecol. 72:631-638.

100.Basu, S., Michaelsson, K., Olofsson, H., Johansson, S., and Melhus, H. 2001. Association between oxidative stress and bone mineral density. Biochem. Biophys. Res. Commun. 288:275-279.

101.Maggio, D., et al. 2003. Marked decrease in plasma antioxidants in aged osteoporotic women: results of a cross-sectional study. J. Clin. Endocrinol. Metab. 88:1523-1527.

102. Muthusami, S., et al. 2005. Ovariectomy induces oxidative stress and impairs bone antioxidant system in adult rats. Clin. Chim. Acta. 360:81-86.

103.Lean, J.M., et al. 2003. A crucial role for thiol antioxidants in estrogen-deficiency bone loss. J. Clin. Invest. 112:915-923. doi:10.1172/JCI200317261.

104.Wimalawansa, S.J., De Marco, G., Gangula, P., and Yallampalli, C. 1996. Nitric oxide donor alleviates ovariectomy-induced bone loss. Bone. 18:301-304.

105.Hao, Y.J., Tang, Y., Chen, F.B., and Pei, F.X. 2005. Different doses of nitric oxide donor prevent osteoporosis in ovariectomized rats. Clin. Orthop. Relat. Res. 435:226-231.

106.Jamal, S.A., Cummings, S.R., and Hawker, G.A. 2004. Isosorbide mononitrate increases bone formation and decreases bone resorption in postmenopausal women: a randomized trial. J. Bone Miner. Res. 19:1512-1517.

107. Steinbeck, M.J., Appel, W.H., Jr., Verhoeven, A.J., and Karnovsky, M.J. 1994. NADPH-oxidase expression and in situ production of superoxide by osteoclasts actively resorbing bone. J. Cell Biol. 126:765-772.

108.Ha, H., et al. 2004. Reactive oxygen species mediate RANK signaling in osteoclasts. Exp. Cell Res. 301:119-127.

109.Lean, J.M., Jagger, C.J., Kirstein, B., Fuller, K., and Chambers, T.J. 2005. Hydrogen peroxide is essential for estrogen-deficiency bone loss and osteoclast formation. Endocrinology. 146:728-735.

110.Reth, M. 2002. Hydrogen peroxide as second messenger in lymphocyte activation. Nat. Immunol. 3:1129-1134.

111.Lean, J., Kirstein, B., Urry, Z., Chambers, T., and Fuller, K. 2004. Thioredoxin-1 mediates osteoclast stimulation by reactive oxygen species. Biochem. Biophys. Res. Commun. 321:845-850.

112.Chen, J.R., et al. 2005. Transient versus sustained phosphorylation and nuclear accumulation of ERKs underlie anti-versus pro-apoptotic effects of estrogens. J. Biol. Chem. 280:4632-4638.

113.Jagger, C.J., Lean, J.M., Davies, J.T., and Chambers, T.J. 2005. Tumor necrosis factor-alpha mediates osteopenia caused by depletion of antioxidants. Endocrinology. 146:113-118.

114.Mizuashi, M., Ohtani, T., Nakagawa, S., and Aiba, S. 2005. Redox imbalance induced by contact sensitizers triggers the maturation of dendritic cells. J. Invest. Dermatol. 124:579-586.

115.Rutault, K., Alderman, C., Chain, B.M., and Katz, D.R. 1999. Reactive oxygen species activate human peripheral blood dendritic cells. Free Radic. Biol. Med. 26:232-238.

116. Maemura, K., et al. 2005. Reactive oxygen species are essential mediators in antigen presentation by Kupffer cells. Immunol. Cell Biol. 83:336-343.

117.Vosters, O., et al. 2003. Dendritic cells exposed to nacystelyn are refractory to maturation and promote the emergence of alloreactive regulatory $\mathrm{T}$ cells. Transplantation. 75:383-389.

118.Verhasselt, V., et al. 1999. N-acetyl-L-cysteine inhibits primary human $\mathrm{T}$ cell responses at the dendritic cell level: association with NF-kappaB inhibition. J. Immunol. 162:2569-2574.

119.Matsue, H., et al. 2003. Generation and function of reactive oxygen species in dendritic cells during antigen presentation. J. Immunol. 171:3010-3018.

120.Hildeman, D.A., et al. 1999. Reactive oxygen species regulate activation-induced $\mathrm{T}$ cell apoptosis. Immunity. 10:735-744.

121.Grassi, F., and Pacifici, R. 2005. Oxidative stress induced dendritic cell-dependent $\mathrm{T}$ cell activation. A novel mechanism by which estrogen deficiency causes bone loss. J. Bone Min. Res. 20:a1144.

122. Elefteriou, F., et al. 2005. Leptin regulation of bone resorption by the sympathetic nervous system and CART. Nature. 434:514-520.

123.Fox, H.S., Bond, B.L., and Parslow, T.G. 1991. Estrogen regulates the IFN-gamma promoter. J. Immunol. 146:4362-4367. 